\title{
New Type of Sturm-Liouville Problems in Associated Hilbert Spaces
}

\author{
O. Sh. Mukhtarov ${ }^{1,2}$ and K. Aydemir ${ }^{1}$ \\ ${ }^{1}$ Department of Mathematics, Faculty of Science, Gaziosmanpaşa University, 60250 Tokat, Turkey \\ ${ }^{2}$ Institute of Mathematics and Mechanics, Azerbaijan National Academy of Sciences, Baku, Azerbaijan \\ Correspondence should be addressed to K. Aydemir; kadriye.aydemir@gop.edu.tr
}

Received 6 November 2013; Accepted 2 January 2014; Published 16 April 2014

Academic Editor: Qingying Bu

Copyright (C) 2014 O. Sh. Mukhtarov and K. Aydemir. This is an open access article distributed under the Creative Commons Attribution License, which permits unrestricted use, distribution, and reproduction in any medium, provided the original work is properly cited.

\begin{abstract}
We introduce a new type of discontinuous Sturm-Liouville problems, involving an abstract linear operator in equation. By suggesting own approaches we define some new Hilbert spaces to establish such properties as isomorphism, coerciveness, and maximal decreasing of resolvent operator with respect to spectral parameter. Then we find sufficient conditions for discreteness of the spectrum and examine asymptotic behaviour of eigenvalues. Obtained results are new even for continuous case, that is, without transmission conditions.
\end{abstract}

\section{Introduction}

Various modifications of classical Sturm-Liouville problems have attracted a lot of attention in the recent past because of the appearance of new important applications in mathematics, mechanics, physics, electronics, geophysics, meteorology, and other branches of natural sciences (see [1-9] and references cited therein). For example, they describe the vibrational modes of various systems, such as the vibrations of a string or the energy eigenfunctions of a quantum mechanical oscillator, in which case the eigenvalues correspond to the resonant frequencies of vibration or energy levels. It was, in part, the idea that the discrete energy levels observed in atomic systems could be obtained as the eigenvalues of a differential operator which led Schrödinger to propose his wave equation. The simplest example of a Sturm-Liouville operator is the constant-coefficient secondderivative operator, whose eigenfunctions are trigonometric functions. Many other important special functions, such as Airy functions and Bessel functions, are associated with variable-coefficient Sturm-Liouville operators. One feature that occurs for Sturm-Liouville operators, which does not occur for matrices, is the possibility of an absolutely continuous spectrum. Instead of eigenfunction expansions, we then get integral transforms, of which the Fourier transform is an example. Other, more complicated spectral phenomena can also occur. For example, eigenvalues embedded in a continuous spectrum, singular continuous spectrum, and pure point spectrum consisting of eigenvalues that are dense in an interval (see [2]).

In this paper we will examine a new type of SturmLiouville equation involving an abstract linear operator $T$, namely, the equation

$$
L u:=-u^{\prime \prime}+q(x) u+\left.T u\right|_{x}=\lambda u
$$

on $[-1,0) \cup(0,1]$, together with eigen-dependent boundary conditions:

$$
\begin{gathered}
b_{1} u:=\alpha_{1} u(-1)+\alpha_{2} u^{\prime}(-1)=0 \\
b_{2} u:=\left(\beta_{1} u(1)-\beta_{2} u^{\prime}(1)\right)+\lambda\left(\beta_{1}^{\prime} u(1)-\beta_{2}^{\prime} u^{\prime}(1)\right)=0
\end{gathered}
$$

and transmission conditions at one interior point $x=0$ :

$$
\begin{gathered}
t_{1} u:=\gamma_{1} u(0-)-\delta_{1} u(0+)=0, \\
t_{2} u:=\gamma_{2} u^{\prime}(0-)-\delta_{2} u^{\prime}(0+)=0,
\end{gathered}
$$

where $\alpha_{i}, \beta_{i}, \beta_{i}^{\prime}, \delta_{i}$, and $\gamma_{i}(i=1,2)$ are real numbers, $q(x)$ is a real-valued function and continuous in each $[-1,0)$ and $(0,1]$ 
which have finite limits $q( \pm 0)=\lim _{x \rightarrow \pm 0} q(x), \lambda$ is a complex spectral parameter, and $T$ is an abstract linear operator (unbounded and non-self-adjoint in general) in Hilbert space $H=L_{2}(-1,0) \oplus L_{2}(0,1)$. Naturally, everywhere we will assume that $\left|\alpha_{1}\right|+\left|\alpha_{2}\right| \neq 0,\left|\beta_{1}\right|+\left|\beta_{2}\right|+\left|\beta_{1}^{\prime}\right|+$ $\left|\beta_{2}^{\prime}\right| \neq 0,\left|\delta_{i}\right|+\left|\gamma_{i}\right| \neq 0$, and $D(T) \subset W_{2}^{2}(-1,0) \oplus W_{2}^{2}(0,1)$. By standard arguments (see [10]) we will also assume that $\rho:=$ $\beta_{1}^{\prime} \beta_{2}-\beta_{2}^{\prime} \beta_{1}>0$. Note that the "Sturm-Liouville" problem studied here is new and nonstandard, since it contains a nondifferential term, namely, an abstract linear operator $T$ in the equation. Moreover, spectral parameter $\lambda$ appears not only in the equation, but also in the boundary conditions and two supplementary transmission conditions are given at one interior point.

Some special cases of this problem arise after an application of the method of separation of variables to the varied assortment of physical problems. For example, some boundary value problems with transmission conditions arise in heat and mass transfer problems [7], in vibrating string problems when the string is loaded additionally with point masses [8], and in diffraction problems [9]. Different challenges emerged with the development of the Sturm theory and a corresponding awareness of the importance of distinguishing the absolutely continuous component from other parts of the essential spectrum, in connection with existence and completeness of the wave operators $[3,11,12]$.

\section{Operator Treatment in Associated Hilbert Spaces}

For operator-theoretic interpretation we will introduce some modified Hilbert spaces according to boundary-transmission conditions. For this throughout in the below we will assume that $\delta_{1} \delta_{2}>0, \gamma_{1} \gamma_{2}>0$ and define modified Hilbert spaces $\widetilde{L}_{2}$ and $H_{1}$ as follows. Firstly, we will replace the standard inner product in direct sum space $L_{2}(-1,0) \oplus L_{2}(0,1)$, which is given by

$$
\langle u, v\rangle_{L_{2}}:=\int_{-1}^{0} u(x) \overline{v(x)} d x+\int_{0}^{1} u(x) \overline{v(x)} d x
$$

for $u, v \in L_{2}(-1,0) \oplus L_{2}(0,1)$, by modified inner product

$$
\langle u, v\rangle_{\widetilde{L}_{2}}:=\gamma_{1} \gamma_{2} \int_{-1}^{0} u(x) \overline{v(x)} d x+\delta_{1} \delta_{2} \int_{0}^{1} u(x) \overline{v(x)} d x .
$$

Then we will replace the standard inner product in the direct sum space $H_{0}=\left(L_{2}(-1,0) \oplus L_{2}(0,1)\right) \oplus \mathbb{C}$, which is given by

$$
\langle U, V\rangle_{H_{0}}:=\langle u(\cdot), v(\cdot)\rangle_{L_{2}}+u_{1} \overline{v_{1}}
$$

for $U=\left(u(\cdot), u_{1}\right), V=\left(v(\cdot), v_{1}\right) \in H_{0}$, by the modified inner product accordance with boundary-transmission conditions as

$$
\langle U, V\rangle_{H_{1}}:=\langle u, v\rangle_{\widetilde{L}_{2}}+\frac{\delta_{1} \delta_{2}}{\rho} u_{1} \overline{v_{1}}
$$

and apply operator theory in the Hilbert space

$$
\left.H_{1}:=\left(L_{2}(-1,0) \oplus L_{2}(0,1)\right) \oplus \mathbb{C},\langle\cdot, \cdot\rangle_{H_{1}}\right) .
$$

Remark 1. It is readily seen that the modified inner product (8) is equivalent to the standard of inner product $\left(L_{2}(-1,0) \oplus\right.$ $\left.L_{2}(0,1)\right) \oplus \mathbb{C}$, so $H_{1}$ is also Hilbert space and can be seen as different realization of the Hilbert space $H_{0}$. But such realization of direct sum space $H_{0}$ allows us to interpret the conditions (2)-(4) as self-adjoint boundary-transmission conditions.

Denoting $\ell(u):=\beta_{1} u(1)-\beta_{2} u^{\prime}(1)$ and $\ell^{\prime}(u):=\beta_{1}^{\prime} u(1)-$ $\beta_{2}^{\prime} u^{\prime}(1)$, define a linear operator $£$ in direct sum space $H_{1}$ by action low:

$$
£\left(u, u_{1}\right):=(L w,-\ell(u))
$$

on the domain of definition $D(\mathfrak{E})$ consisting of all $U=$ $\left(u(\cdot), u_{1}\right) \in H_{1}$ which satisfy the following conditions:

(i) $u(x)$ and $u^{\prime}(x)$ are absolutely continuous on both $[-1,-\delta)$ and $(\delta, 1]$ for arbitrary $\delta>0$ and have finite limits $u(\mp 0)$ and $u^{\prime}(\mp 0)$,

(ii) $L u \in L_{2}(-1,0) \oplus L_{2}(0,1)$,

(iii) $b_{1}(u)=t_{1}(u)=t_{2}(u)=0$,

(iv) $u_{1}=\ell^{\prime}(u)$.

Consequently we can reformulate the boundary valuetransmission problem (BVTP) (1)-(4) in the operatorequation form as

$$
£ U=\lambda U
$$

in the Hilbert space $H_{1}$.

Lemma 2. The linear operator $£$ is densely defined in $H_{1}$.

Proof. It is enough to prove that, if $G_{0}=\left(g_{0}(\cdot), g_{1}\right) \in H_{1}$ is orthogonal to all $F=\left(f(\cdot), \ell^{\prime}(f)\right) \in D(\mathfrak{E})$, then $G_{0}=(0,0)$. Suppose that

$$
\left\langle F, G_{0}\right\rangle_{H_{1}}=0 \quad \forall F \in D(\mathfrak{E}) .
$$

Denote by $C_{0}^{\infty}[-1,0) \oplus C_{0}^{\infty}(0,1]$ the set of infinitely differentiable functions in $[-1,0) \cup(0,1]$, each of which vanishes on some neighborhoods of the points $x=-1, x=0$, and $x=1$. Since $(f(\cdot), 0) \in D(\mathfrak{E})$ for each $f \in C_{0}^{\infty}[-1,0) \oplus C_{0}^{\infty}(0,1]$, we have from (12) that

$$
\gamma_{1} \gamma_{2} \int_{-1}^{0} f(x) \overline{g_{0}(x)} d x+\delta_{1} \delta_{2} \int_{0}^{1} f(x) \overline{g_{0}(x)} d x=0
$$

for all $f \in C_{0}^{\infty}[-1,0) \oplus C_{0}^{\infty}(0,1]$ which in turn implies that

$$
\int_{-1}^{0} f(x) \overline{g_{0}(x)} d x=\int_{0}^{1} f(x) \overline{g_{0}(x)} d x=0
$$

for all $f \in C_{0}^{\infty}[-1,0) \oplus C_{0}^{\infty}(0,1]$. Taking into account that $C_{0}^{\infty}[-1,0)$ and $C_{0}^{\infty}(0,1]$ are dense in $L_{2}(-1,0)$ and $L_{2}(0,1)$, respectively, we have that the function $g_{0}(x)$ vanishes on $[-1,0) \cup(0,1]$. Furthermore, by choosing an element $F_{0}=$ $\left(f_{0}(\cdot), \ell^{\prime}\left(f_{0}\right)\right) \in D(\mathfrak{E})$ such that $\ell^{\prime}\left(f_{0}\right)=1$ and putting in (12) we have $g_{1}=0$. Hence $G_{0}=(0,0)$. The proof is complete. 
Now let $\mathfrak{E}_{0}$ be linear differential operator in Hilbert space $H_{1}$ with domain $D\left(\mathfrak{E}_{0}\right)=D(\mathfrak{E})$ and action low:

$$
\mathfrak{E}_{0}\left(f(x), \ell^{\prime}(f)\right)=\left(-f^{\prime \prime}+q(x) f,-\ell(f)\right) .
$$

Lemma 3. The operator $\mathfrak{E}_{0}$ is symmetric in $H_{1}$.

Proof. Let $u\left(u(x), u_{1}\right), v=\left(v(x), v_{1}\right) \in D(\mathfrak{E})$ be any two elements. Integrating twice by parts, we have

$$
\begin{aligned}
\left\langle £_{0} U, V\right\rangle_{H_{1}}= & \left\langle U, £_{0} V\right\rangle_{H_{1}}+\gamma_{1} \gamma_{2} W(u, \bar{v} ;-0) \\
& -\gamma_{1} \gamma_{2} W(u, \bar{v} ;-1)+\delta_{1} \delta_{2} W(u, \bar{v} ; 1) \\
& -\delta_{1} \delta_{2} W(u, \bar{v} ;+0) \\
& +\frac{\delta_{1} \delta_{2}}{\rho}\left(\ell^{\prime}(u) \ell(\bar{v})-\ell(u) \ell^{\prime}(\bar{v})\right),
\end{aligned}
$$

where $W(u, v ; x):=u(x) v^{\prime}(x)-u^{\prime}(x) v(x)$. From the fact, that $u$ and $\bar{v}$ satisfied the first boundary condition (2) we have $W(u, \bar{v} ;-1)=0$ in turn; since $u$ and $\bar{v}$ satisfies both transmission conditions (4) it follows that

$$
\delta_{1} \delta_{2} W(u, \bar{v} ;+0)=\gamma_{1} \gamma_{2} W(u, \bar{v} ;-0)
$$

Further, the direct calculations give

$$
\ell^{\prime}(u) \ell(\bar{v})-\ell(u) \ell^{\prime}(\bar{v})=-\rho W(u, \bar{v} ; 1)
$$

Now, putting these equalities in (16) we have needed equality

$$
\left\langle\mathfrak{E}_{0} U, V\right\rangle_{H_{1}}=\left\langle U, \mathfrak{E}_{0} V\right\rangle_{H_{1}}
$$

Corollary 4. The eigenvalues of $\mathfrak{E}_{0}$ are real.

Theorem 5. $\mathfrak{E}_{0}$ is self-adjoint linear operator in the Hilbert space $H_{1}$.

Proof. For shortening denote $\tau u:=-u^{\prime \prime}+q(x) u$. Since $\mathfrak{E}_{0}$ are symmetric and densely defined in $H_{1}$ it is enough to show that if $\left\langle\mathfrak{E}_{0} U, V\right\rangle_{H_{1}}=\langle U, W\rangle_{H_{1}}$ for all $U=\left(u, \ell^{\prime}(u)\right) \in D\left(\mathfrak{E}_{0}\right)$ then $V \in D\left(\mathfrak{E}_{0}\right)$ and $\mathfrak{E}_{0} V=W$ where $V=(v(x), h)$ and $W=(w(x), k)$; that is, (i) $v, v^{\prime} \in A C_{\mathrm{loc}}((-1,0)), v, v^{\prime} \in$ $A C_{\mathrm{loc}}((0,1))$, and $\tau v \in H_{1}$; (ii) $h=\ell^{\prime}(v)=\beta_{1}^{\prime} v(1)-\beta_{2}^{\prime} v^{\prime}(1)$; (iii) $b_{1} v=t_{1} v=t_{2} v=0$; (iv) $w(x)=\tau v$; and (v) $k=-\ell(v)=$ $-\beta_{1} v(1)+\beta_{2} v^{\prime}(1)$. For all $U \in\left(C_{0}^{\infty}[-1,0) \oplus C_{0}^{\infty}(0,1]\right) \oplus 0 \subset$ $D\left(\mathfrak{E}_{0}\right)$, we find that $\langle\tau u, v\rangle_{L_{2}}=\langle u, w\rangle_{L_{2}}$. Hence, by standard Sturm-Liouville theory, (i) and (iv) hold. By (iv), the equation $\left\langle\mathfrak{E}_{0} U, V\right\rangle_{H_{1}}=\langle U, W\rangle_{H_{1}}$ for all $U \in D\left(\mathfrak{E}_{0}\right)$ becomes

$$
\langle\tau u, v\rangle_{\widetilde{L}_{2}}=\langle u, \tau v\rangle_{\widetilde{L}_{2}}+\frac{\delta_{1} \delta_{2}}{\rho} \ell^{\prime}(u) \bar{k}+\frac{\delta_{1} \delta_{2}}{\rho} \ell(u) \bar{h} .
$$

On the other hand, by two partial integrations we get

$$
\begin{aligned}
\langle\tau u, v\rangle_{\tilde{L}_{2}}= & \gamma_{1} \gamma_{2} \int_{-1}^{0}\left(-u^{\prime \prime}+q(x) u\right) \bar{v} d x \\
& +\delta_{1} \delta_{2} \int_{0}^{1}\left(-u^{\prime \prime}+q(x) u\right) \bar{v} d x \\
= & \langle u, \tau v\rangle_{\tilde{L}_{2}}+\gamma_{1} \gamma_{2} W(u, \bar{v} ;-0) \\
& -\gamma_{1} \gamma_{2} W(u, \bar{v} ;-1)+\delta_{1} \delta_{2} W(u, \bar{v} ; 1) \\
& -\delta_{1} \delta_{2} W(u, \bar{v} ;+0) .
\end{aligned}
$$

Therefore,

$$
\begin{aligned}
& \frac{\delta_{1} \delta_{2}}{\rho} \ell^{\prime}(u) \bar{k}+\frac{\delta_{1} \delta_{2}}{\rho} \ell(u) \bar{h} \\
& =\gamma_{1} \gamma_{2} W(u, \bar{v} ;-0)-\gamma_{1} \gamma_{2} W(u, \bar{v} ;-1) \\
& \quad+\delta_{1} \delta_{2} W(u, \bar{v} ; 1)-\delta_{1} \delta_{2} W(u, \bar{v} ;+0) .
\end{aligned}
$$

By Naimark's patching lemma [13] there is $U_{1}=\left(u_{1}, \ell^{\prime}\left(u_{1}\right)\right) \epsilon$ $D\left(\mathfrak{E}_{0}\right)$ such that $u_{1}(-1)=u_{1}^{\prime}(-1)=u_{1}(0-)=u_{1}^{\prime}(0-)=$ $u_{1}(0+)=u_{1}^{\prime}(0+)=0, u_{1}(1)=\beta_{2}^{\prime}$ and $u_{1}^{\prime}(1)=\beta_{1}^{\prime}$. Putting $U=U_{1}$ in (22) we conclude that $h=\beta_{1}^{\prime} v(1)-\beta_{2}^{\prime} v^{\prime}(1)$. Thus, (ii) holds. Similarly, we can prove (v). It remains to show that (iii) holds. Choose $U_{2} \in D\left(\mathfrak{E}_{0}\right)$ so that $u_{2}(1)=$ $u_{2}^{\prime}(1)=u_{2}(0-)=u_{2}^{\prime}(0-)=0, u_{2}(1)=\alpha_{2}$ and $u_{2}^{\prime}(1)=$ $-\alpha_{1}$. Then $\ell^{\prime}\left(u_{2}\right)=\ell\left(u_{2}\right)=0$. Putting in (22), we obtain $\alpha_{1} v(-1)+\alpha_{2} v^{\prime}(-1)=0$. Let $U_{3} \in D\left(\mathfrak{E}_{0}\right)$ satisfy $u_{3}(1)=$ $u_{3}^{\prime}(1)=u_{3}(0+)=u_{3}(0-)=0, u_{3}^{\prime}(0-)=\gamma_{1} / \delta_{1}$ and $u_{3}^{\prime}(0+)=\delta_{1} \delta_{2} / \gamma_{1} \gamma_{2}$. Then, $\ell\left(u_{3}\right)=\ell^{\prime}\left(u_{3}\right)=0$. By (22) we get $v(0+)=\left(\gamma_{1} / \delta_{1}\right) v(0-)$. Lastly, choose $U_{4} \in D\left(£_{0}\right)$ so that $u_{4}(1)=u_{4}^{\prime}(1)=u_{4}(-1)=u_{4}^{\prime}(-1)=u_{4}^{\prime}(0+)=u_{4}^{\prime}(0-)=0$, $u_{4}(0-)=\gamma_{2} / \delta_{2}$ and $u_{4}(0+)=\delta_{1} \delta_{2} / \gamma_{1} \gamma_{2}$. In this case $\ell\left(u_{4}\right)=$ $\ell^{\prime}\left(u_{4}\right)=0$. Putting in $(22)$ we get $v^{\prime}(0+)=\left(\gamma_{2} / \delta_{2}\right) v^{\prime}(0-)$. Thus $V \in D\left(\mathfrak{E}_{0}\right)$. The proof is complete.

\section{Maximal Decreasing of the Resolvent Operator and Discreteness of the Spectrum}

To establish the topological isomorphism and coerciveness we need to introduce a new inner product space $\mathrm{H}_{2}$ as linear space:

$$
\begin{gathered}
\left\{U=\left(u(\cdot), u_{1}\right): u(\cdot) \in W_{2}^{2}(-1,0) \oplus W_{2}^{2}(0,1),\right. \\
\left.b_{1}(u)=t_{1}(u)=t_{2}(u)=0, u_{1}=\ell^{\prime}(u)\right\},
\end{gathered}
$$

equipped with the inner product

$$
\left\langle\left(u(\cdot), u_{1}\right),\left(v(\cdot), v_{1}\right)\right\rangle_{H_{2}}=\langle u(\cdot), v(\cdot)\rangle_{W_{2}^{2}} .
$$

It can be verified easily that all axioms of inner product are satisfied.

Lemma 6. $\mathrm{H}_{2}$ is a Hilbert space. 
Proof. Let $U_{n}=\left(u_{n}(\cdot), \ell^{\prime}\left(u_{n}\right)\right), n=1,2, \ldots$, be any Cauchy sequence in $H_{2}$. Then by $(24)$ the sequence $\left(u_{n}(\cdot)\right)$, which consists of the first components of $\left(U_{n}\right)$, will be a Cauchy sequence in the Hilbert space $W_{2}^{2}(-1,0) \oplus W_{2}^{2}(0,1)$ and therefore is convergent in this space. Let $w=w(\cdot) \epsilon$ $W_{2}^{2}(-1,0) \oplus W_{2}^{2}(0,1)$ be limit of this sequence. By virtue of the fact that the embeddings $W_{2}^{2}(-1,0) \subset C[-1,0]$ and $W_{2}^{2}(0,1) \subset C[0,1]$ are continuous, the sequences $b_{1}\left(u_{n}\right)$, $t_{1}\left(u_{n}\right)$, and $t_{2}\left(u_{n}\right)$ are converges to $b_{1}(w), t_{1}(w)$, and $t_{2}(w)$, accordingly. Hence $b_{1}(w)=t_{1}(w)=t_{2}(w)=0$ since $b_{1}\left(u_{n}\right)=t_{1}\left(u_{n}\right)=t_{2}\left(u_{n}\right)=0$ for all $n$ by (23). Now, defining $U_{0}=\left(u(\cdot), \ell^{\prime}(w)\right)$ we see that $U_{0} \in H_{2}$ and the sequence $\left(U_{n}\right)$ converges to $U_{0}$ in $H_{2}$; so, the arbitrary Cauchy sequence in $\mathrm{H}_{2}$ is convergent. The proof is complete.

Theorem 7. If the operator $T$ is compact from $W_{2}^{2}(-1,0) \oplus$ $W_{2}^{2}(0,1)$ into $L_{2}(-1,0) \oplus L_{2}(0,1)$ then, for any $\varepsilon>0$, there exists $R_{\varepsilon}>0$ such that for all complex numbers $\lambda$ satisfying $\varepsilon<\arg \lambda<2 \pi-\varepsilon,|\lambda|>R_{\varepsilon}$, the operator $£-\lambda I$ is an isomorphism from $\mathrm{H}_{2}$ onto $H_{1}$ and for these $\lambda$ the coercive estimate

$$
\|U(\lambda, F)\|_{H_{2}}+|\lambda|\|U(\lambda, F)\|_{H_{1}} \leq C(\varepsilon)\|F\|_{H_{1}}
$$

holds for the solution $U=U(\lambda, F)$ of the equation $(\lambda I-£) U=$ $F, F \in H_{1}$, where $C(\varepsilon)$ is a constant, which depends only on $\varepsilon$.

Proof. It is obvious that the linear operator $\lambda I-£$ acts from $\mathrm{H}_{2}$ into $H_{1}$ continuously for all $\lambda \in \mathbb{C}$. Furthermore, proceeding in a similar manner as in [14], we obtain that for any $\varepsilon>0$ there exists $R_{\varepsilon}>0$ such that for all complex numbers $\lambda$ satisfying $\varepsilon<\arg \lambda<2 \pi-\varepsilon,|\lambda|>R_{\varepsilon}$, the operator $\widetilde{L}(\lambda): u \rightarrow\left(\lambda u-L(\lambda) u, \lambda \ell^{\prime}(u)+\ell(u)\right)$ from $W_{2}^{2}(-1,0) \oplus W_{2}^{2}(0,1)$ onto $\left(L_{2}(-1,0) \oplus L_{2}(0,1)\right) \oplus \mathbb{C}$ is an isomorphism and for these $\lambda$ the coercive estimate

$$
\|u\|_{W_{2}^{2}}+|\lambda|\left(\|u\|_{L_{2}}+\left|\ell^{\prime}(u)\right|\right) \leq C(\varepsilon)\left(\|f\|_{L_{2}}+\left|f_{1}\right|\right)
$$

holds for a solution $u(x)$ of the problem $L u-\lambda u=f, b_{1}(u)=$ $0, b_{2}(u)=f_{1}$, and $t_{1}(u)=t_{2}(u)=0$, where $F=\left(f(\cdot), f_{1}\right) \in$ $H_{1}$. Consequently, the operator $£-\lambda I$ is an isomorphism from $H_{2}$ onto $H_{1}$. The estimate (25) follows from (26).

Definition 8 (see [9]). Let $A$ be densely defined closed operator in complex Hilbert space $E$. The point $\lambda$ of the complex plane is called a regular point of an operator $A$ in $E$, if the operator $A-\lambda I$ is invertible (i.e., has a bounded inverse operator $R(\lambda, A)=(A-\lambda I)^{-1}$ which is defined on whole $E$ ). In this case the operator $R(\lambda, A)=(A-\lambda I)^{-1}$ is called the resolvent of the operator $A$. The complement of the set of regular points $\rho(A)$ to the entire complex plane is called the spectrum $\sigma(A)$ of the operator (obviously, all eigenvalues belong to the spectrum).

Corollary 9. From the coercive estimate (25), in particular, it follows that the maximal decreasing of the resolvent operator $R(\lambda, £)=(£-\lambda I)^{-1}$, namely, the estimate

$$
\|R(\lambda, £)\|_{H_{1} \rightarrow H_{1}} \leq C(\varepsilon)|\lambda|^{-1},
$$

holds for all complex $\lambda$ as in the formulation of the last Theorem.

Definition 10 (see [12]). Let $\lambda_{0}$ be eigenvalue of $A$. The linear manifold

$$
N_{\lambda_{0}}=U_{n=1}^{\infty}\left\{u \in E: u \in D\left(A^{n}\right),\left(A-\lambda_{0} I\right)^{n} u=0\right\}
$$

is called a root lineal corresponding to eigenvalue $\lambda_{0}$. The dimension of the lineal $N_{\lambda_{0}}$ is called an algebraic multiplicity of the eigenvalue $\lambda_{0}$. The spectrum $\sigma(A)$ of the operator $A$ is called discrete if $\sigma(A)$ consist of isolated eigenvalues with finite algebraic multiplicities and infinity is the only possible limit point of $\sigma(A)$.

Theorem 11. If the operator $T$ acts compactly from $W_{2}^{2}(-1,0) \oplus$ $W_{2}^{2}(0,1)$ into $L_{2}(-1,0) \oplus L_{2}(0,1)$, then the spectrum of the problem (1)-(4) is discrete.

Proof. At first show that the embedding $H_{2} \subset H_{1}$ is compact. For this, let $U_{n}=\left(u_{n}(\cdot), \ell^{\prime}\left(u_{n}\right)\right), n=1,2, \ldots$, be any bounded sequence in $\mathrm{H}_{2}$. Then the sequence $\left(u_{n}(\cdot)\right)$ consisting of the first components of $\left(U_{n}\right)$ will be bounded in the direct sum space $W_{2}^{2}(-1,0) \oplus W_{2}^{2}(0,1)$. Since the embeddings $W_{2}^{2}(-1,0) \subset L_{2}[-1,0]$ and $W_{2}^{2}(0,1) \subset L_{2}[0,1]$ are compact, the sequence $\left(u_{n}(\cdot)\right)$ has a convergent subsequence $\left(u_{n_{k}}(\cdot)\right)$ in the space $L_{2}(-1,0) \oplus L_{2}(0,1)$. Let $u_{0}(\cdot) \in L_{2}(-1,0) \oplus$ $L_{2}(0,1)$ be limit of this subsequence. Further, since the embedding $W_{2}^{2}(0,1) \subset C[0,1]$ is compact, the sequence $\left(u_{n_{k}}(\cdot)\right)$ has a convergent subsequence $\left(u_{n_{k_{s}}}(\cdot)\right)$ in space $C[0,1]$. Consequently the numerical sequence $\left(\ell^{\prime}\left(u_{n_{k_{s}}}\right)\right)$ is convergent. Let $u_{1} \in \mathbb{C}$ be limit of this numerical sequence. Now defining $U_{0}=\left(u_{0}(\cdot), u_{1}\right)$, we see that $U_{0} \in H_{1}$ and the sequence $\left(U_{n_{k_{s}}}\right)$ converges to $U_{0}$ in the Hilbert space $H_{1}$, so the embedding $H_{2} \subset H_{1}$ is compact. Further, from the coercive estimate (25) in particular, it follows that the resolvent operator $R(\lambda, \mathfrak{E})$ acts boundedly from $H_{1}$ into $H_{2}$. Consequently, the resolvent operator $R(\lambda, \mathfrak{E})$ acts compactly from $H_{1}$ into itself. Then by virtue of well-known theorem of functional analysis (see, e.g., ([11], Chapter III, Section 6)) the spectrum of $£$ is discrete.

\section{Distribution of the Eigenvalues in the Complex Plane}

Define a linear operator $\widetilde{T}$ in the Hilbert space $H_{1}$ with domain $D(\widetilde{T})=D(\mathfrak{E})$ and action low:

$$
\widetilde{T}(F)=(T f, 0)
$$

for $F=\left(f(x), f_{1}\right) \in D(\widetilde{T})$. Then, the considered problem (1) $-(4)$ can be written in the operator-equation form as

$$
\left(\mathfrak{E}_{0}+\widetilde{T}\right) U=\lambda U, \quad U \in D(\mathfrak{E}) .
$$

Remark 12. The eigenvalues of the problems (1)-(4) and (30) coincide, and the corresponding eigenfunctions of (1)-(4) coincide with the first components of the corresponding eigenelements of $\mathfrak{E}=\mathfrak{E}_{0}+\widetilde{T}$. 
Let $A$ be densely defined closed operator in complex Hilbert space $H$ and let $\mathbb{G}$ be any subset of complex plane $\mathbb{C}$ and $r>0$ any real number. By $N(r, \mathbb{G}, A)$ we will denote the number of eigenvalues of $A$ belonging to $\mathbb{G}$, which are smaller than $r$ and are counted according to their algebraic multiplicity.

Definition 13 (see [15]). Let $A_{1}$ be any closed linear operator having at least one regular point. A linear (in general, unbounded) operator $A_{2}$ is said to be $A_{1}$-compact if $D\left(A_{2}\right) \supseteq$ $D\left(A_{1}\right)$ and if for some regular point $\lambda_{0} \in \rho\left(A_{1}\right)$ the operator $A_{2} R\left(\lambda_{0}, A_{1}\right)=A_{2}\left(A_{1}-\lambda_{0} I\right)^{-1}$ is compact. in [15].

The following theorem can be deduced from Theorem 3.2

Theorem 14. Let $S$ be self-adjoint operator in Hilbert space the spectrum $\sigma(C)$ of which is discrete, $T$ be S-compact operator, and $A=S+T$. Then if $S$ has precisely denumerable many positive eigenvalues and

$$
N\left(r(1+\varepsilon), R^{+}, S\right) \sim N\left(r, R^{+}, S\right), \quad r \longrightarrow \infty, \varepsilon \longrightarrow 0,
$$

then for any $\alpha(0<\alpha<\pi / 2)$

$$
N\left(r, \mathbb{G}_{\alpha}, A\right) \sim N\left(r, R^{+}, S\right), \quad r \longrightarrow \infty,
$$

where $R^{+}=(0, \infty), \mathbb{G}_{\alpha}:=\{\lambda \in \mathbb{C} \mid \alpha<\arg \lambda<$ $2 \pi-\alpha\}$, and $f(\lambda) \sim g(\lambda)$ as $r \rightarrow \infty$ is the abbreviation for $\lim _{r \rightarrow \infty} f(r) / g(r)=1$.

Lemma 15. The operator $£_{0}$ has precisely denumerable many eigenvalues $\mu_{n}, n=0,1,2$, with the asymptotic representation

$$
\mu_{n}=\frac{\pi^{2} n^{2}}{4}+O(n)
$$

Proof. Let $\mu \in \mathbb{C}$ and let $\varphi(x, \mu)=\left\{\begin{array}{l}\varphi_{1}(x, \mu), x \in[-1,0) \\ \varphi_{2}(x, \mu), x \in(0,1]\end{array}\right.$ be solution of the equation $-u^{\prime \prime}+q(x) u=\mu u$ for which $\varphi_{1}(-1, \mu)=\alpha_{2}$, $\varphi_{1}^{\prime}(-1, \mu)=-\alpha_{1}, \varphi_{2}(+0, \mu)=\gamma_{1} \delta_{1}^{-1} \varphi_{1}(-0, \mu)$, and $\varphi_{2}^{\prime}(+0, \mu)=$ $\gamma_{2} \delta_{2}^{-1} \varphi_{1}^{\prime}(-0, \mu)$. Obviously, this solution satisfies the first boundary condition (2) and both transmission conditions (4). Consequently, eigenvalues of $\mathfrak{E}_{0}$ coincide with the zeros of the entire function

$$
\begin{aligned}
\Delta(\mu)= & b_{2}(\varphi(\cdot, \mu)) \\
= & \mu\left(\beta_{1}^{\prime} \varphi_{2}(1, \mu)-\beta_{2}^{\prime} \varphi_{2}^{\prime}(1, \mu)\right) \\
& +\left(\beta_{1} \varphi_{2}(1, \mu)-\beta_{2} \varphi_{2}^{\prime}(1, \mu)\right) .
\end{aligned}
$$

Consider the case $\alpha_{2} \beta_{2}^{\prime} \neq 0$. By proceeding with the same procedure as in [16] we have

$$
\begin{aligned}
\varphi_{2}^{(k)}(x, \mu)= & \alpha_{2}\left[\gamma_{1} \delta_{1}^{-1} \cos \sqrt{\mu}(\cos \sqrt{\mu} x)^{(k)}\right. \\
& \left.-\gamma_{2} \delta_{2}^{-1} \sin \sqrt{\mu}(\sin \sqrt{\mu} x)^{(k)}\right] \\
& +O\left(|\sqrt{\mu}|^{k-1} e^{|\operatorname{Im} \mu|(x+1)}\right) .
\end{aligned}
$$

Putting in previous equality we get

$$
\Delta(\mu)=\frac{\alpha_{2} \beta_{2}^{\prime}\left(\delta_{1}+\delta_{2}\right)}{2 \delta_{1} \delta_{2}}(\sqrt{\mu})^{3} \sin 2 \sqrt{\mu}+O\left(|\sqrt{\mu}|^{2} e^{2|\operatorname{Im} \mu|}\right) .
$$

Now by applying the well-known Rouche's theorem (see, e.g., [13]) we can prove that the function $\Delta(\mu)$ has precisely denumerable many zeros $\mu_{n}, n=0,1,2, \ldots$, with the asymptotic representation

$$
\mu_{n}=\frac{\pi^{2} n^{2}}{4}+O(n), \quad n \longrightarrow \infty
$$

The proof is complete.

Lemma 16. Let the operator $T$ be compact with respect to $£_{0}$ in the Hilbert space $H_{1}$. Then

(i) the spectrum of $£=\mathfrak{E}_{0}+\widetilde{T}$ is discrete and consists of precisely denumerable many eigenvalues;

(ii) for any arbitrary small $\alpha>0$, all eigenvalues of $£$ with the possible exception of a finite number lie in the sector $\psi_{\alpha}=\{\lambda \in \mathbb{C}:|\arg \lambda|<\alpha\}$ of angular $2 \alpha$;

(iii) for the sequence of eigenvalues $\left(\lambda_{n, \alpha}\right), n \geq 0$ belongs to the sector $\psi_{\alpha}$, which, when listed according to nondecreasing modulus and repeated according to algebraic multiplicity, the asymptotic formula

$$
\left|\lambda_{n, \alpha}\right|=\frac{\pi^{2} n^{2}}{4}+o\left(n^{2}\right), \quad n \longrightarrow \infty,
$$

holds.

Proof. From asymptotic formula (33) it follows that

$$
\frac{\pi^{2} n^{2}}{4}+c_{1} n \leq \mu_{n} \leq \frac{\pi^{2} n^{2}}{4}+c_{2} n
$$

for some real numbers $c_{1}$ and $c_{2}$. In turn, from this we can easily derive that

$$
N,\left(r, R^{+}, \mathfrak{E}_{0}\right)=\frac{2 \sqrt{r}}{\pi}+O\left(\frac{1}{\sqrt{r}}\right), \quad r \longrightarrow \infty .
$$

Consequently

$$
\lim _{\substack{r \rightarrow \infty \\ \varepsilon \rightarrow 0}} \frac{N\left(r(1+\varepsilon), R^{+}, \mathfrak{E}_{0}\right)}{N\left(r, R^{+}, \mathfrak{E}_{0}\right)}=1 .
$$

Taking into account the above, we have that, for any small $\alpha>0$,

$$
\begin{aligned}
N\left(r, \mathbb{G}_{\alpha}, \mathfrak{E}\right) & =N\left(r, R^{+}, \mathfrak{E}_{0}\right)+o\left(N\left(r, R^{+}, \mathfrak{E}_{0}\right)\right) \\
& =\frac{2 \sqrt{r}}{\pi}+o(\sqrt{r}) \quad \text { as } r \longrightarrow \infty,
\end{aligned}
$$

where, as usual, the expression $f(r)=o(g(r)), r \rightarrow \infty$, is the abbreviation for $\lim _{r \rightarrow \infty} f(r) / g(r)=0$. Putting $r=\left|\lambda_{n, \alpha}\right|$ in the last equality we have

$$
\left|\lambda_{n, \alpha}\right|=\frac{\pi^{2} n^{2}}{4}+o\left(\left|\lambda_{n, \alpha}\right|\right) \quad \text { as } n \longrightarrow \infty .
$$


From that it immediately follows that

$$
\left|\lambda_{n, \alpha}\right|=\frac{\pi^{2} n^{2}}{4}+o\left(n^{2}\right), \quad n \longrightarrow \infty
$$

The proof is complete.

Theorem 17. Under conditions of previous lemma the spectrum $\sigma(£)$ of the operator $£$ is discrete and consists of denumerable many eigenvalues $\lambda_{n}, n=0,1,2 \ldots$, which, when arranged in nondecreasing modulus and counted to their algebraic multiplicity, the asymptotic formulas

$$
\begin{gathered}
\operatorname{Re} \lambda_{n}(£)=\frac{\pi^{2} n^{2}}{4}+o\left(n^{2}\right), \\
\operatorname{Im} \lambda_{n}(£)=o\left(n^{2}\right), \quad n \longrightarrow \infty
\end{gathered}
$$

hold.

Proof. Taking in view that for all small $\alpha>0$ there are at most finite number eigenvalues of $£$ outside the angle $\mathbb{G}_{\alpha}$, from Lemma 16 it follows that

$$
\left|\lambda_{n, \alpha}\right|=\frac{\pi^{2} n^{2}}{4}+o\left(n^{2}\right), \quad n \longrightarrow \infty .
$$

Again, by Theorem 7 for all $\alpha>0$, small enough, there are $n_{\alpha}$ such that for all $n \geq n_{\alpha}$ the inequalities $\operatorname{Re} \lambda_{n}>\left|\lambda_{n}\right| \cos \alpha$ and $\left|\operatorname{Im} \lambda_{n}\right|<\left|\lambda_{n}\right| \sin \alpha$ hold. Letting $\alpha \rightarrow \infty$ we have that

$$
\operatorname{Re} \lambda_{n} \sim\left|\lambda_{n}\right|, \quad\left|\operatorname{Im} \lambda_{n}\right|=o\left(\left|\lambda_{n}\right|\right), \quad n \longrightarrow \infty .
$$

Combining with (46) yields the needed formulas

$$
\operatorname{Re} \lambda_{n}(£)=\frac{\pi^{2} n^{2}}{4}+o\left(n^{2}\right), \quad\left|\operatorname{Im} \lambda_{n}\right|=o\left(n^{2}\right), \quad n \rightarrow \infty .
$$

The main result of this section is the following theorem.

Theorem 18. Let the operator $T$ be acted compactly from $W_{2}^{2}(-1,0) \oplus W_{2}^{2}(0,1)$ into $L_{2}(-1,0) \oplus L_{2}(0,1)$. Then, the spectrum of BVTP (1)-(4) is discrete and consists of precisely denumerable many eigenvalues $\lambda_{n}, n=1,2, \ldots$, which, when listed according to decreasing real part and repeated according to algebraic multiplicity, has the following asymptotic representation:

$$
\lambda_{n}=\frac{\pi^{2} n^{2}}{4}+o\left(n^{2}\right) \quad \text { as } n \longrightarrow \infty
$$

Proof. By virtue of Theorem 11 the resolvent $R(\lambda, \mathfrak{E})$ acted boundedly from $H_{1}$ to $H_{2}$. On the other hand, the operator $\widetilde{T}$, defined by (29), acted compactly from $H_{2}$ to $H_{1}$, by assumption on $\mathrm{T}$ and definition of $\mathrm{H}_{2}$. Consequently the operator $\widetilde{T} R(\lambda, £)$ is compact in the Hilbert space $H_{1}$; that

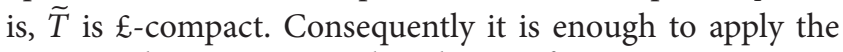
previous theorem to complete the proof.

\section{Examples}

Let us give some examples of abstract linear operator $T$ as follows:

$$
\text { (1) } T u=\sum_{i=1}^{n}\left(a_{i}(x) u\left(c_{i}\right)+b_{i}(x) u^{\prime}\left(d_{i}\right)\right) \text {, }
$$

where the functions $a_{i}(x)$ and $b_{i}(x)$ satisfy the same conditions as $q(x) ; c_{i}, d_{i} \in(-1,0) \cup(0,1)$ are interior points;

$$
\text { (2) } \begin{aligned}
T u= & \int_{-1}^{0} G_{10}(x, s) u(s) d s \\
& +\int_{-1}^{0} G_{11}(x, s) u^{\prime}(s) d s \\
& +\int_{0}^{1} G_{20}(x, s) u(s) d s+\int_{0}^{1} G_{21}(x, s) u^{\prime}(s) d s,
\end{aligned}
$$

where the Kernels $G_{1 i}(x, s)$ and $G_{2 i}(x, s)(i=1,2)$ are defined and continuous in $[-1,1] \times[-1,0]$ and $[-1,1] \times[0,1]$, respectively.

Consequently, the results of this study can be applied to the wide variety class of boundary value problems.

\section{Concluding Remarks}

All results in this study are derived under condition $\delta_{1} \delta_{2}>0$ and $\gamma_{1} \gamma_{2}>0$. Let us show that this simple condition cannot be omitted. For this, consider the following simple special case of the problem (1)-(4) for which the condition $\delta_{1} \delta_{2}>0$ does not hold:

$$
\begin{gathered}
-u^{\prime \prime}=\lambda u, \quad x \in[-1,0) \cup(0,1], \\
u(-1)=0, \quad \lambda u(1)=u^{\prime}(1), \\
u(-0)=u(+0), \quad u^{\prime}(-0)=-u^{\prime}(+0) .
\end{gathered}
$$

We can show that this problem has only the trivial solution $u=0$ for any $\lambda \in \mathbb{C}$. Thus, if $\delta_{1} \delta_{2}<0$ then the spectrum of the problem (52) may be empty. Moreover, it is well known that for the standard Sturm-Liouville problems the eigenvalues are real and the first asymptotic term has the form $O(n)$. But for our problem, the eigenvalues may be also nonreal complex numbers and the asymptotic term appears in the "weak" form as $o\left(n^{2}\right)$ because of the abstract linear operator $T$ in the equation.

\section{Conflict of Interests}

The authors declare that there is no conflict of interests regarding the publication of this paper.

\section{References}

[1] Z. Akdoğan, M. Demirci, and O. S. Mukhtarov, "Discontinuous Sturm-Liouville problems with eigenparameter-dependent boundary and transmissions conditions," Acta Applicandae Mathematicae, vol. 86, no. 3, pp. 329-344, 2005. 
[2] P. W. Anderson, "Absence of diffusion in certain random lattices," Physical Review, vol. 109, no. 5, pp. 1492-1505, 1958.

[3] W. O. Amrein and V. Georgescu, "Strong asymptotic completeness of wave operators for highly singular potentials," Helvetica Physica Acta, vol. 47, pp. 517-533, 1974.

[4] E. Uğurlu and E. Bairamov, "On singular dissipative fourthorder differential operator in Lim-4 case," ISRN Mathematical Analysis, vol. 2013, Article ID 549876, 5 pages, 2013.

[5] W. Kerner, Computing the Complex Eigenvalue Spectrum for Resistive Magnetohydrodynamics, Elsevier Science, NorthHolland, 1986.

[6] M. Kobayashi, "Eigenvalues of discontinuous Sturm-Liouville problems with symmetric potentials," Computers and Mathematics with Applications, vol. 18, no. 4, pp. 357-364, 1989.

[7] A. V. Likov and A. Yu. Mikhailov, The Theory of Heat and Mass Transfer, Qosenergaizdat, 1963, (Russian).

[8] A. N. Tikhonov and A. A. Samarskii, Equations of Mathematical Physics, Pergamon, New York, NY, USA, 1963.

[9] N. N. Voitovich, B. Z. Katsenelbaum, and A. N. Sivov, Generalized Method of Eigen-Vibration in the Theory of Diffraction, Nakua, Mockow, Russia, 1997, (Russian).

[10] J. Walter, "Regular eigenvalue problems with eigenvalue parameter in the boundary condition," Mathematische Zeitschrift, vol. 133, no. 4, pp. 301-312, 1973.

[11] T. Kato, Perturbation Theory for Linear Operators, Springer, Berlin, Germany, 1984.

[12] S. Y. Yakubov, Completeness of Root Functions of Regular Differential Operators, Longman, New York, NY, USA, 1994.

[13] E. C. Titchmarsh, Eigenfunctions Expansion Associated with Second Order Differential Equations I, Oxford University Press, Oxford, UK, 2nd edition, 1962.

[14] O. Sh. Muhtarov and S. Yakubov, "Problems for ordinary differential equations with transmission conditions," Applicable Analysis, vol. 81, no. 5, pp. 1033-1064, 2002.

[15] A. S. Markus and V. I. Matsaev, "Comparison theorems for spectra of linear operators and spectral asymptotics," Trudy Moskovskogo Matematicheskogo Obshchestva, vol. 45, pp. 133181, 1982.

[16] K. Aydemir and O. Sh. Mukhtarov, “Green's function method for self-adjoint realization of boundary-value problems with interior singularities," Abstract and Applied Analysis, vol. 2013, Article ID 503267, 7 pages, 2013. 


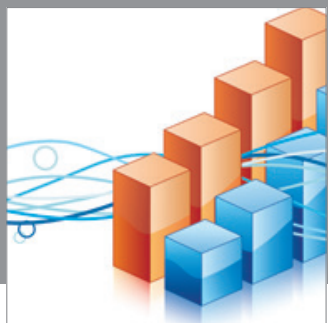

Advances in

Operations Research

mansans

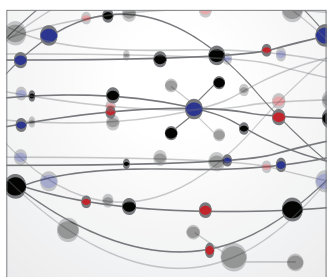

The Scientific World Journal
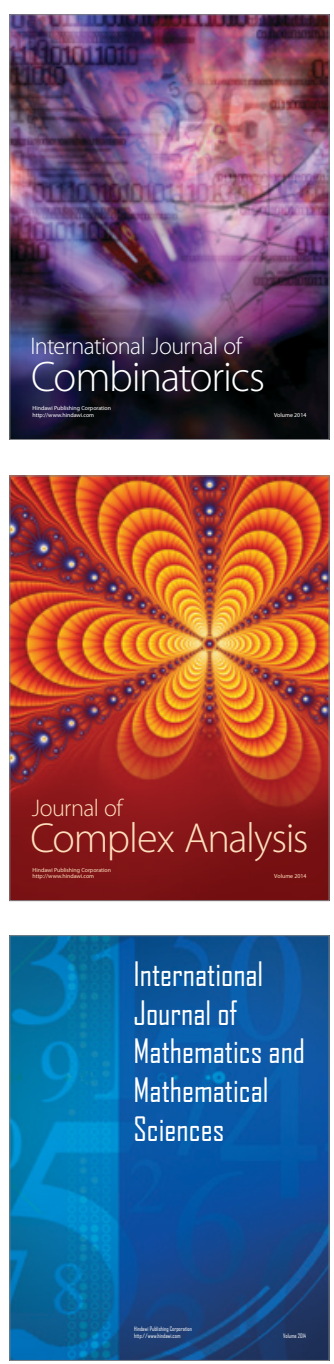
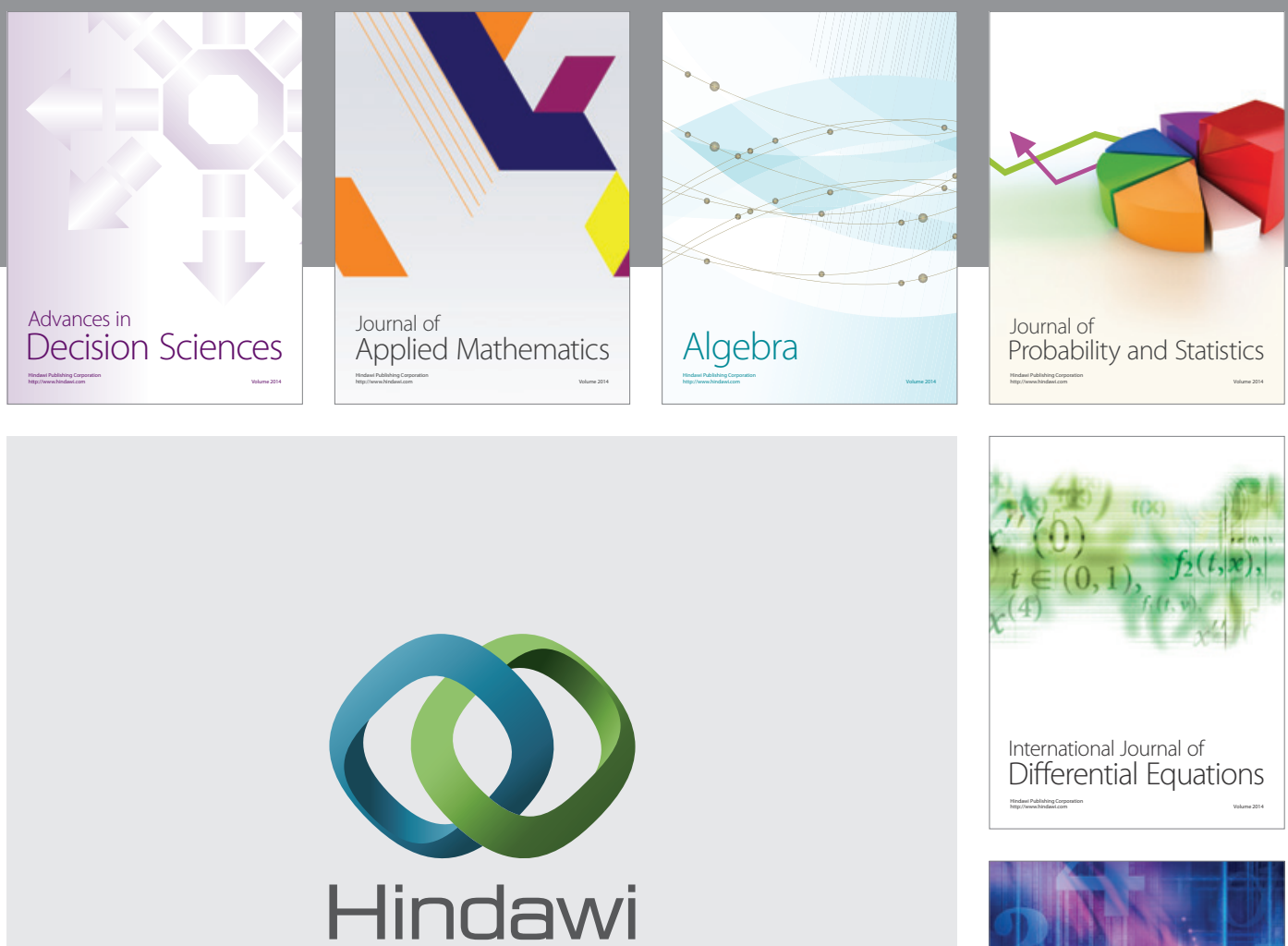

Submit your manuscripts at http://www.hindawi.com
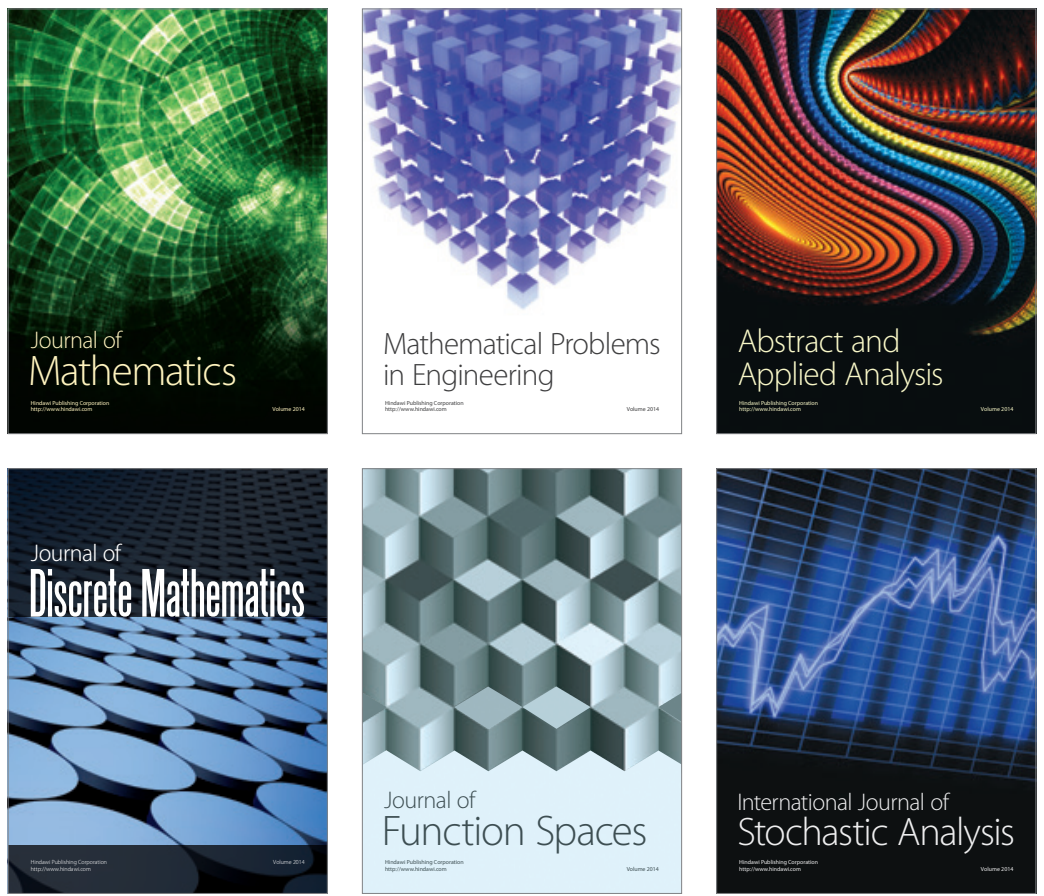

Journal of

Function Spaces

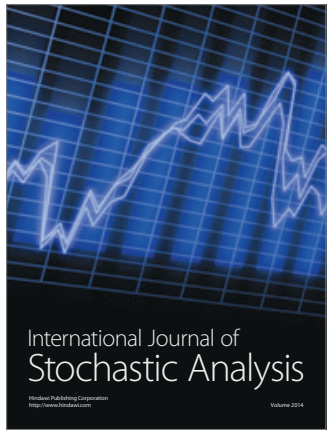

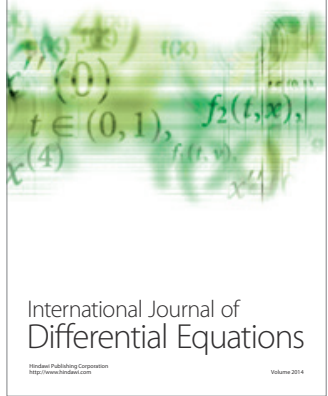
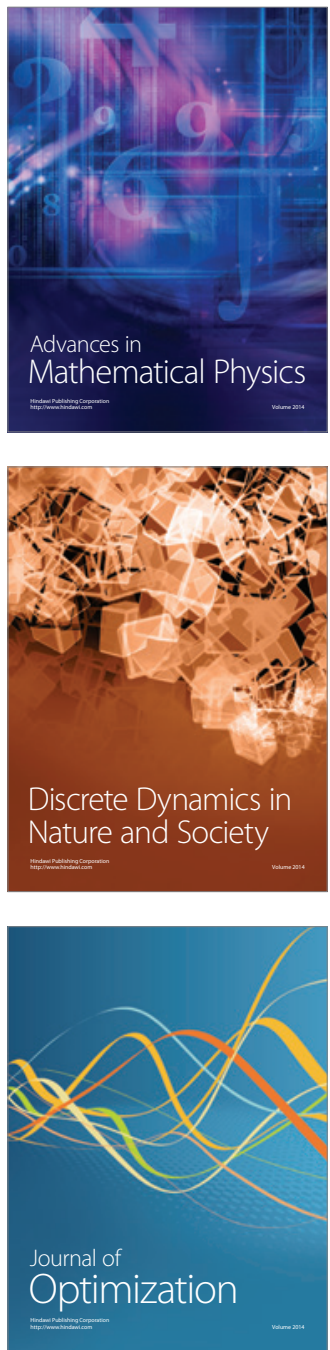